\title{
GGTase-I deficiency reduces tumor formation and improves survival in mice with K-RAS-induced lung cancer
}

\author{
Anna-Karin M. Sjogren, ${ }^{1}$ Karin M.E. Andersson, ${ }^{1}$ Meng Liu, ${ }^{1,2}$ Briony A. Cutts, ${ }^{1}$ \\ Christin Karlsson, ${ }^{1}$ Annika M. Wahlstrom, ${ }^{1}$ Martin Dalin, ${ }^{1}$ Carolyn Weinbaum, ${ }^{3}$ \\ Patrick J. Casey, ${ }^{3}$ Andrej Tarkowski, ${ }^{4}$ Birgitta Swolin, ${ }^{5}$ Stephen G. Young, ${ }^{6}$ and Martin O. Bergo ${ }^{1}$ \\ ${ }^{1}$ Wallenberg Laboratory, Institute of Medicine, Sahlgrenska University Hospital, Göteborg, Sweden. ${ }^{2}$ Department of Neurosurgery, Qilu Hospital, \\ Shandong University, Jinan, People's Republic of China. ${ }^{3}$ Department of Pharmacology and Cancer Biology, Duke University Medical Center, \\ Durham, North Carolina, USA. ${ }^{4}$ Department of Rheumatology and Inflammation Research and ${ }^{5}$ Department of Clinical Chemistry and \\ Transfusion Medicine, Institute of Medicine, Sahlgrenska University Hospital, Göteborg, Sweden. ${ }^{6}$ Department of Medicine, \\ Division of Cardiology, David Geffen School of Medicine, UCLA, Los Angeles, California, USA.
}

\begin{abstract}
Protein geranylgeranyltransferase type I (GGTase-I) is responsible for the posttranslational lipidation of CAAX proteins such as RHOA, RAC1, and cell division cycle 42 (CDC42). Inhibition of GGTase-I has been suggested as a strategy to treat cancer and a host of other diseases. Although several GGTase-I inhibitors (GGTIs) have been synthesized, they have very different properties, and the effects of GGTIs and GGTase-I deficiency are unclear. One concern is that inhibiting GGTase-I might lead to severe toxicity. In this study, we determined the effects of GGTase-I deficiency on cell viability and K-RAS-induced cancer development in mice. Inactivating the gene for the critical $\beta$ subunit of GGTase-I eliminated GGTase-I activity, disrupted the actin cytoskeleton, reduced cell migration, and blocked the proliferation of fibroblasts expressing oncogenic K-RAS. Moreover, the absence of GGTase-I activity reduced lung tumor formation, eliminated myeloproliferative phenotypes, and increased survival of mice in which expression of oncogenic K-RAS was switched on in lung cells and myeloid cells. Interestingly, several cell types remained viable in the absence of GGTase-I, and myelopoiesis appeared to function normally. These findings suggest that inhibiting GGTase-I may be a useful strategy to treat K-RAS-induced malignancies.
\end{abstract}

\section{Introduction}

More than 100 intracellular proteins contain a CAAX motif that directs isoprenylation at a carboxyterminal cysteine (the " $C$ " of the CAAX motif) (1). Some CAAX proteins, such as RHOA, cell division cycle 42 (CDC42), and RAP1, are geranylgeranylated by protein geranylgeranyltransferase type I (GGTase-I). Others, such as K-RAS and N-RAS, are farnesylated by protein farnesyltransferase (FTase) (2). If the " $X$ " of the CAAX motif is a leucine, the protein is generally geranylgeranylated; otherwise, it is farnesylated (3). Isoprenylation renders the carboxyl terminus of the CAAX proteins more hydrophobic, enhancing their ability to bind to membranes within cells, and also regulates protein-protein interactions. GGTase-I and FTase share a common $\alpha$ subunit but have unique $\beta$ subunits that dictate their substrate specificities (2).

In some eukaryotic cells, GGTase-I is an essential enzyme. Null mutations in the $\beta$ subunit of GGTase-I are lethal in both Drosophila melanogaster (4) and Saccharomyces cerevisiae (5). The lethality of GGTase-I deficiency in S. cerevisiae was likely due to the failure to geranylgeranylate Rho1 $\mathrm{p}$ and $\mathrm{Cdc} 42 \mathrm{p}$, as the lethal-

Nonstandard abbreviations used: AAH, atypical adenomatous hyperplasia; CDC42, cell division cycle 42; fCDC42, farnesylated CDC42; fRHOA, farnesylated RHOA; FTase, farnesyltransferase; FTI, FTase inhibitor; GGTase-I, geranylgeranyltransferase type I; GGTI, GGTase-I inhibitor; $K^{L S L}$, Kras2 $2^{L S L}$; LC, lysozyme M-Cre; MAC-2, galectin3; MAC-3, sialoadhesin; Pggt $1 b^{f l}$ mice, mice with a conditional knockout allele for the $\beta$ subunit of GGTase-I.

Conflict of interest: S.G. Young received consulting and speaker fees from Isis Pharmaceuticals and Merck.

Citation for this article: J. Clin. Invest. 117:1294-1304 (2007). doi:10.1172/JCI30868. ity could be overcome by expressing mutant Rho1p and Cdc42p proteins engineered to undergo farnesylation by FTase (6). Interestingly, Candida albicans and Arabidopsis thaliana are viable in the absence of GGTase-I $(7,8)$. Remarkably, the impact of GGTase-I deficiency in mammalian cells has never been studied.

The realization that the RAS proteins are farnesylated (9) has fueled interest in protein isoprenylation. Farnesylation is important for the proper membrane targeting of RAS proteins and for their transforming ability (10). In mouse models, farnesyltransferase inhibitors (FTIs) have significant antitumor activity and minimal toxicity (11). In human clinical trials, however, FTIs have been disappointing, at least for the treatment of solid tumors (12), likely because K-RAS and N-RAS - the RAS isoforms most often implicated in human cancer - can be geranylgeranylated in the presence of an FTI (13).

The realization that K-RAS and N-RAS can be geranylgeranylated in the setting of FTI therapy prompted efforts to develop GGTase-I inhibitors (GGTIs). GGTIs could be used in combination with FTIs to block the isoprenylation of K-RAS and N-RAS. The rationale for using GGTIs in cancer therapy is further underscored by the fact that geranylgeranylated proteins, such as RHOA, RHOC, and RALA, are intimately involved in tumor development and metastasis (14-16). Several GGTIs inhibit the growth of tumor cell lines, including cell lines with K-RAS mutations (17-19). Interest in developing GGTIs actually extends beyond cancer therapy (20). Inhibition of GGTase-I ameliorated disease phenotypes in a mouse model of multiple sclerosis (21) and inhibited hepatitis $\mathrm{C}$ viral replication in hepatoma cells 
A

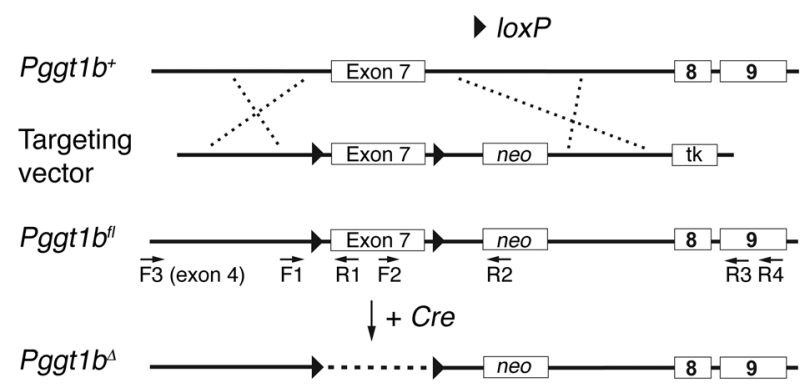

B Starting Pggt1b genotype: $\mathrm{fl} /+\quad \mathrm{fl} / \mathrm{fl}$ Adenovirus treatment: $\beta$-gal Cre $\beta$-gal Cre Resulting genotype: $\mathrm{fl} /+\Delta /+\mathrm{fl} / \mathrm{fl} \quad \Delta / \Delta$
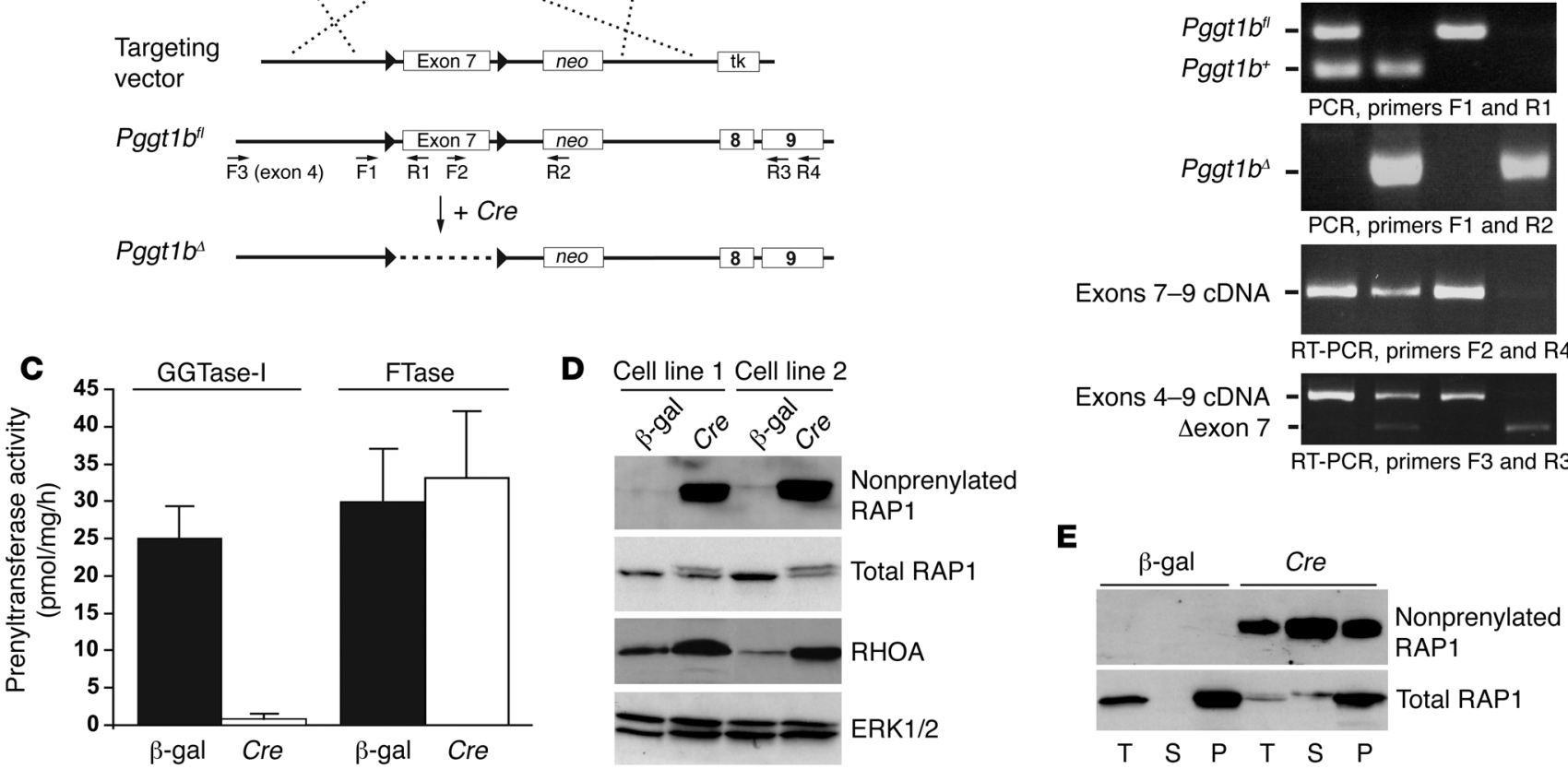

D Cell line 1 Cell line 2

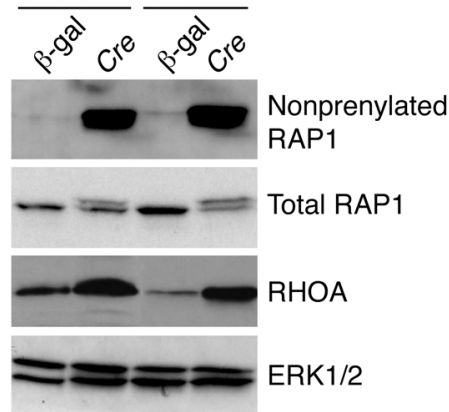

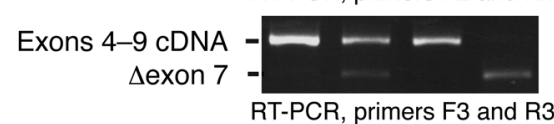

$\mathbf{E}$

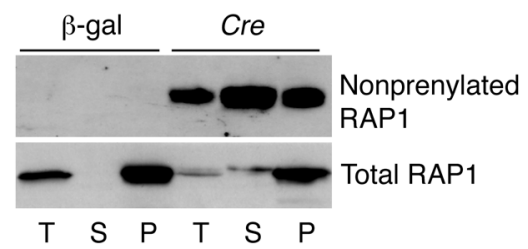

Figure 1

Generation of a conditional knockout allele for the $\beta$ subunit of GGTase-I $\left(P g g t 1 b^{f}\right)$. (A) A sequence-replacement gene-targeting vector in which exon 7 of Pggt1b is flanked by loxP sites (arrowheads). Expression of Cre recombinase results in the excision of exon 7, creating a frameshift mutation and a null allele. The locations of primers for genotyping are indicated. neo, neomycin phosphotransferase cassette; tk, thymidine kinase. (B) PCR and RT-PCR analyses demonstrating the Cre-induced inactivation of Pggt1b. Heterozygous Pggt1 $\mathrm{b}^{\mathrm{fl}+}$ and homozygous Pggt1 $\mathrm{b}^{\mathrm{fl} / \mathrm{fl}}$ fibroblasts were treated with either a $\beta$-gal- or a Cre-adenovirus, and genomic DNA and total RNA were isolated 2 days later. (C) GGTase-I

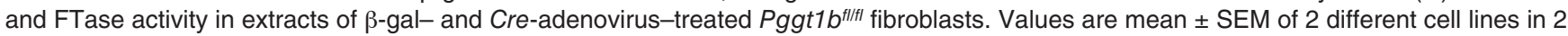
independent experiments. (D) Western blots of extracts from 2 different Pggt1 $b^{f / f t}$ fibroblast cell lines treated with $\beta$-gal-and Cre-adenovirus. The blots were incubated with antibodies recognizing nonprenylated RAP1, total RAP1, and RHOA. Total ERK1/2 was used as a loading control. (E) Distribution of nonprenylated RAP1 and total RAP1 proteins in the membrane (pellet [P]) and cytosolic (soluble [S]) fractions of $\beta$-gal- or Cre-adenovirus-treated Pggt $1 \mathrm{~b}^{f / f t}$ fibroblasts. T, total extract.

(22). Also, inhibiting GGTase-I in C. albicans is being evaluated as a strategy to treat nosocomial infections (23).

Despite indications that inhibiting GGTase-I might be useful therapeutically, there is concern regarding the potential toxicity of this approach, in part because geranylgeranylated proteins are more numerous than farnesylated proteins (1). Another reason for concern is that GGTIs have been shown to induce apoptosis of cultured cells and cause toxicity in mouse models (24-26). Other studies, however, have suggested that some GGTIs might not be particularly toxic $(27,28)$.

We reasoned that our understanding of the physiologic importance of protein geranylgeranylation could be improved substantially with genetic studies in mice. In this study, we created mice with a conditional knockout allele for the $\beta$ subunit of GGTase-I (Pggt $1 b^{f l}$ mice) and defined the impact of Pggt $1 b$ deficiency on cell viability, cell proliferation, and K-RAS-induced oncogenic transformation.

\section{Results}

Generation and validation of a conditional GGTase-I knockout allele. The strategy for creating Pggt $1 b^{f l}$ mice is illustrated in Figure 1A. In the mutant allele (Pggt $\left.1 b^{f l}\right)$, loxP sites flank exon 7 , which is critical for enzymatic activity (29). Pggt $1 b^{f l /+}$ and Pggt $1 b^{f l / f l}$ mice were healthy and fertile. To test the conditional allele, we treated $P g g t 1 b^{f l / f l}$ embryonic fibroblasts with a Cre-adenovirus. Cre recombination converted the Pggt $1 b^{f l}$ allele to a null allele $\left(P g g t 1 b^{\Delta}\right)$, which produced a transcript lacking exon 7 (which yields a frameshift) (Figure 1B). As expected, inactivation of Pggt $1 b$ eliminated GGTase-I activity but did not affect FTase activity (Figure 1C). In heterozygous knockout cells (Pggt $\left.1 b^{\Delta /+}\right)$, GGTase-I activity was reduced by approximately $50 \%$ (data not shown).

To assess the impact of Pggt $1 b$ deficiency on the processing of 2 GGTase-I substrates, RAP1 and RHOA, we subjected fibroblast extracts to Western blot analysis with an antibody specific for nonprenylated RAP1. Nonprenylated RAP1 accumulated in both the soluble and membrane fractions of the Pggt $1 b^{\Delta / \Delta}$ cells (Figure 1, $\mathrm{D}$ and $\mathrm{E})$. Western blots with an antibody recognizing total RAP1 revealed that RAP1 had a slower electrophoretic mobility in Pggt $1 b^{\Delta / \Delta}$ cells (Figure 1, D and E). The inactivation of Pggt1b also resulted in a 2- to 4-fold accumulation of RHOA within cells (Figure 1D).

Pggt1b deficiency blocks proliferation and migration of normal fibroblasts and $K-R A S^{G 12 D}$-expressing fibroblasts but does not affect cell viability. Inactivating a single Pggt $1 b$ allele in Pggt $1 b^{f /+}$ fibroblasts did not affect cell proliferation (Figure 2A). Inactivating both alleles in $P g g t 1 b^{f l / f l}$ cells arrested proliferation (Figure 2A) but had little or no 
A

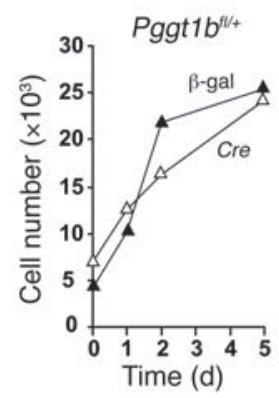

C

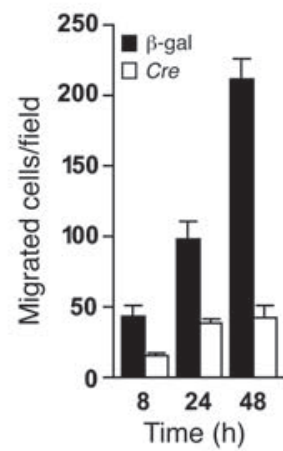

E

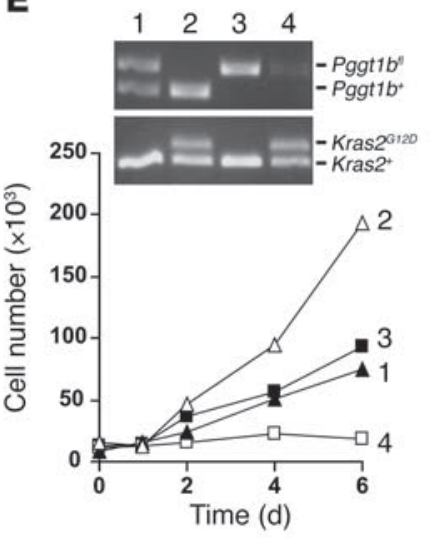

B

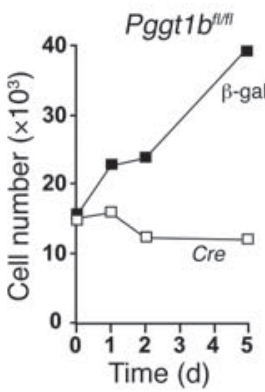

Pggt1
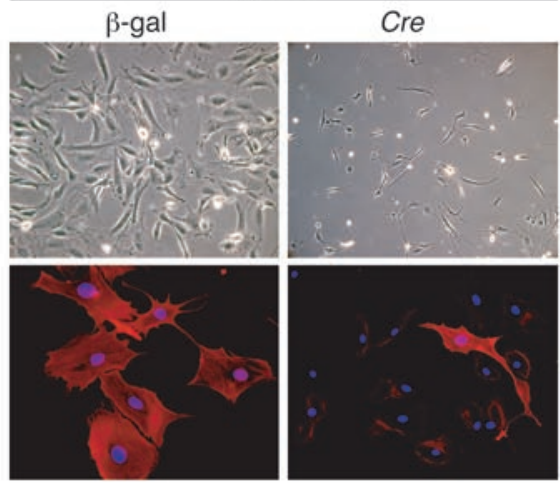

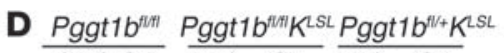

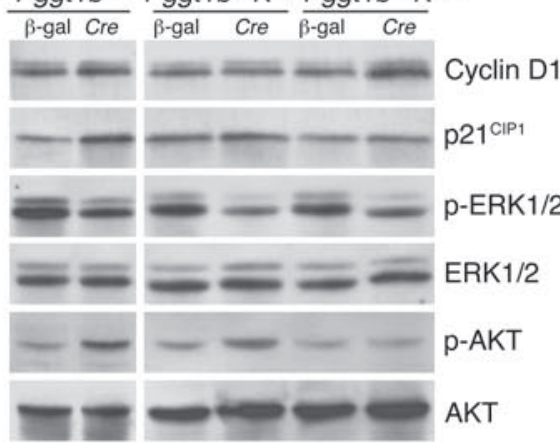

F

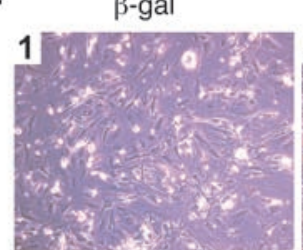

$P g g t 1 b^{1 /+} K^{2 S L}$

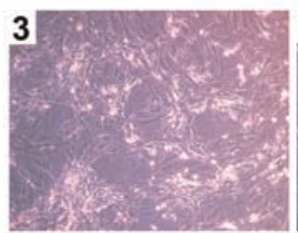

Pggt $1 b^{t m} K^{L S L}$
Cre

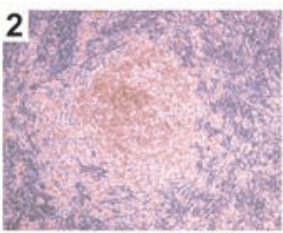

$P_{g g t 1 b^{N+} K^{G 120}}$

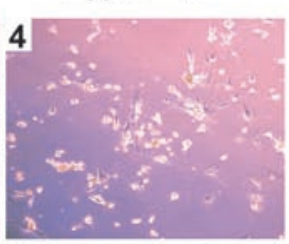

$P_{g g t 1 b^{N A} K^{G 12 D}}$
G

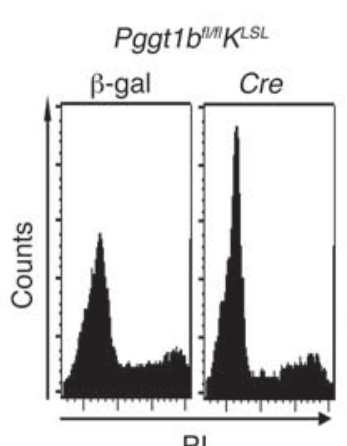

PI

\section{Figure 2}

Inactivation of $P g g t 1 b$ results in proliferation arrest in normal and K-RASG12D_expressing fibroblasts. (A) Effect of $P g g t 1 b$ deficiency on the proliferation of primary mouse fibroblasts. Equal numbers of $\beta$-gal-and Cre-adenovirus-treated primary Pggt $1 \mathrm{~b}^{f / /+}$ and $P g g t 1 b^{f / f / l}$ fibroblasts were seeded on 12-well plates. On days $0,1,2$, and 5 , the cells were trypsinized and counted. Values are mean of 1 cell line assayed in duplicate. Similar results were obtained in 2 experiments with 2 different Pggt $1 b^{f / /+}$ and Pggt $1 b^{f / / t I}$ cell lines. (B) Upper panels: $\beta$-gal-and Cre-adenovirus-treated Pggt $1 b^{f l / t I}$ cells from the experiment described in $\mathbf{A}$. Lower panels: cells from the experiment in $\mathbf{A}$ stained with phalloidin (red) to visualize polymerized actin and with DAPI (blue) to visualize DNA. Original magnification, $\times 10$ (top); $\times 63$ (bottom). (C) Migration of $\beta$-gal- and Cre-adenovirus-treated fibroblasts. Values are mean \pm SEM of 2 cell lines assayed in triplicate. $P<0.01$ at 8 and 24 hours, $P<0.0001$ at 48 hours compared with $\beta$-gal. (D) Western blot of extracts from $\beta$-gal- and Cre-adenovirus-treated primary Pggt1 $b^{f / f t l}, P g g t 1 b^{f / f t} K^{L S L}$, and Pggt1 $b^{f /+}+K^{L S L}$ fibroblasts. (E) Effect of Pggt1b deficiency on the proliferation of K-RASG12D-expressing primary fibroblasts. Equal numbers of $\beta$-gal-and Cre-adenovirus-treated primary $P g g t 1 b^{f /+} K^{L S L}$ and $P g g t 1 b^{f / f f} K^{L S L}$ fibroblasts were seeded in 12 -well plates. On days $0,1,2,4$, and 6 , the cells were trypsinized and counted. Inset shows genotypes of cells assessed by PCR amplification of genomic DNA (lane 1, Pggt1 $b^{f l /+} K^{L S L}$; lane 2, Pggt1 $b^{\Delta /+} K^{G 12 D}$; lane 3, Pggt1 bfl/t/KLSL; lane $\left.4, P g g t 1 b^{\Delta / \Delta} K^{G 12 D}\right)$. Values are mean of 1 cell line assayed in duplicate. Similar results were obtained in 2 experiments with 2 cell lines per genotype. (F) Photographs of fibroblasts from the experiment described in E. Original magnification, $\times 10$. (G) Cell-cycle analysis of $\beta$-gal- and Cre-adenovirus-treated Pggt $1 b^{f / l f I} K^{L S L}$ fibroblasts. The experiment was repeated twice with similar results. PI, propidium iodide.

effect on cell viability, as the cells remained viable for more than 3 weeks after the Cre-adenovirus treatment. The proliferation arrest in $P g g t 1 b^{\Delta / \Delta}$ cells appeared to be irreversible, as we were unable to generate stable $P g g t 1 b^{\Delta / \Delta}$ cell lines. In contrast, we had no difficulty 
A

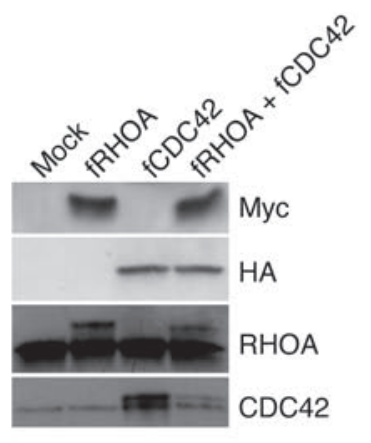

B

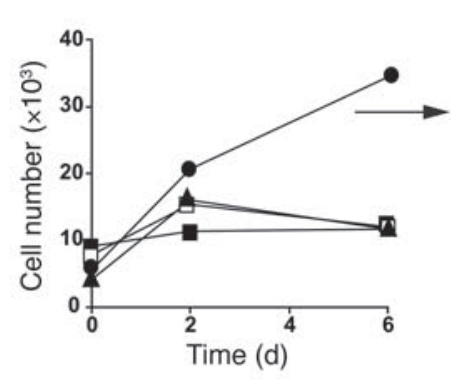

C
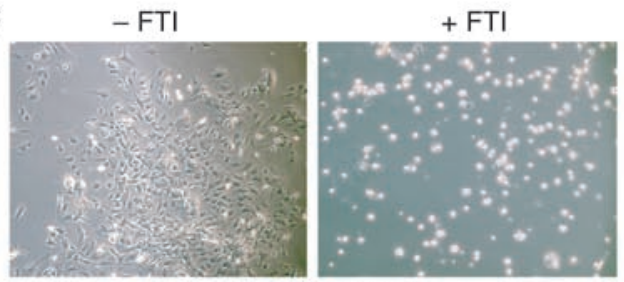

$P g g t 1 b^{N \Delta} K^{G 120}$

$\mathrm{fRHOA}+\mathrm{fCDC} 42$

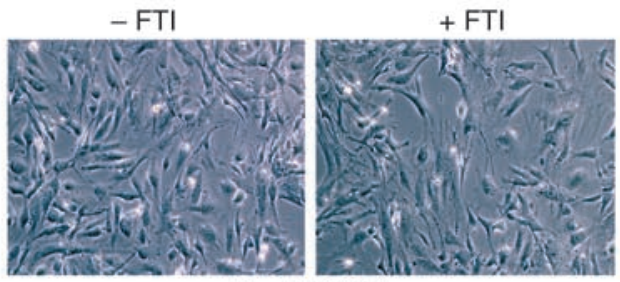

Pggt $1 b^{\text {hII }} K^{L S L}$

\section{Figure 3}

fRHOA and fCDC42 temporarily increase cell proliferation of Pggt1b-deficient K-RAS ${ }^{\mathrm{G} 12 \mathrm{D}}$-expressing fibroblasts. (A) Western blots showing expression of the Myc tag in fRHOA-transfected fibroblasts and the HA tag in fCDC42-transfected fibroblasts. Identical blots were incubated with antibodies recognizing RHOA and CDC42. Note the slower electrophoretic mobility of the tagged proteins. (B) Proliferation of Cre-adenovirustreated primary $P g g t 1 b^{f / f I} K^{L S L}$ fibroblasts transiently transfected with empty plasmid (filled squares) or plasmids encoding Myc-tagged fRHOA (open squares), HA-tagged fCDC42 (filled triangles) or both fRHOA and fCDC42 (black circles). Values are the means of triplicate measurements. The experiment was repeated 3 times with similar results. Transfection of wild-type fibroblasts with these plasmids did not affect cell proliferation. (C) Upper panels show photos of $P g g t 1 b^{f l / f l} K L S L$ fibroblasts transiently transfected with the plasmid encoding fRHOA and fCDC42 and then treated with the Cre-adenovirus. Eight days later, the cells were treated with vehicle (-FTI) or $10 \mu \mathrm{M} \mathrm{FTI-276} \mathrm{(+FTI)} \mathrm{for} 72$ hours. Lower panels show that cells not treated with the Cre-adenovirus were unaffected by the FTI.

isolating Pggt $1 b^{\Delta /+}$ fibroblast cell lines from littermate embryos. The Pggt $1 b^{\Delta / \Delta}$ cells were small and spindle shaped, and the amount of polymerized actin was dramatically reduced (Figure $2 \mathrm{~B}$ ). To determine whether the reduced levels of polymerized actin in $P g g t 1 b^{\Delta / \Delta}$ cells impaired cellular migration, we performed a standard wound closure assay in cultured cells. The ability of Pggt $1 b^{\Delta / \Delta}$ cells to migrate was reduced by $60 \%-80 \%$ (Figure $2 \mathrm{C}$ ). Also, Western blots revealed increased levels of $\mathrm{p} 21^{\mathrm{CIP} 1}$ and phosphorylated $\mathrm{AKT}^{\mathrm{Ser} 473}$ and decreased levels of phosphorylated ERK $1 / 2$ in $P g g t 1 b^{\Delta / \Delta}$ cells than in the parental Pggt $1 b^{f l / f l}$ cells (Figure 2D).

To assess the effect of Pggt1b deficiency on K-RAS-induced cell transformation, we generated primary fibroblasts from Pggt $1 b^{f /+}{ }^{\prime \prime}$ ras $2^{L S L}\left(P g g t 1 b^{f /+} K^{L S L}\right)$ and Pggt $1 b^{f / f l} K^{L S L}$ embryos. The $K^{L S L}$ allele is normally silent; expression of Cre removes a floxed transcriptional terminator sequence (stop cassette) and activates the expression of K-RASG12D (30). Cre-adenovirus treatment of Pggt $1 b^{f l /+} K^{L S L}$ fibroblasts activated K-RAS ${ }^{\mathrm{G} 12 \mathrm{D}}$ expression and resulted in rapid cell proliferation, immortalization, focus formation, increased expression of cyclin D1, and reduced expression of phosphorylated ERK1/2 (Figure 2, D-F). In contrast, Cre-adenovirus treatment of Pggt $1 b^{f l / f l} K^{L S L}$ cells caused cell rounding and proliferation arrest (Figure 2, E and F) but not apoptosis or cell death. Inactivation of Pggt $1 b$ also prevent-

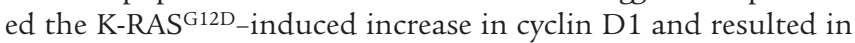
increased levels of phosphorylated AKT but did not affect p21 $1^{\text {CIP1 }}$ levels (Figure 2D). The proliferation arrest in Cre-adenovirus-treated Pggt $1 b^{f l / f l} K^{L S L}$ cells was associated with an increased fraction of cells in the $\mathrm{G}_{1}$ phase of the cell cycle (Figure $2 \mathrm{G}$ ).

Farnesylation of RHOA and CDC42 prevents cell rounding and temporarily restores proliferation of Pggt1b-deficient fibroblasts expressing $K-R A S^{G 12 D}$. In $S$. cerevisiae, expression of farnesylated versions of RHOA and CDC42 (fRHOA and fCDC42, respectively) overcame the lethality associated with a deficiency of the yeast ortholog of Pggt1b (also known as CAL1) (6). Accordingly, we hypothesized that $\mathrm{RHHOA}$ and fCDC42 might overcome the proliferation arrest in Cre-adenovirus-treated $P g g t 1 b^{f l / f l} K^{L S L}$ cells. To test this hypothesis, we transfected primary $P g g t 1 b^{f l / f l} K^{L S L}$ fibroblasts with plasmids encoding fRHOA (Myc-tagged at the amino terminus), fCDC42 (HA-tagged), or both and treated the cells with Creadenovirus. Both $\mathrm{RHOA}$ and $\mathrm{fCDC} 42$ were expressed in transiently transfected cells (Figure $3 \mathrm{~A}$ ). The expression of fRHOA and fCDC42 (but neither construct alone) allowed Pggt $1 b^{\Delta / \Delta} K^{G 12 D}$ cells to escape, at least temporarily, the proliferation arrest and form colonies (Figure 3, B and C). Treatment of the colonies with an FTI promptly induced cell rounding and proliferation arrest (compare Figure 3C with the lower-right panel in Figure 2F). However, even in the absence of an FTI, Pggt $1 b^{\Delta / \Delta} K^{G 12 D}$ colonies eventually stopped growing and could not be developed into stable cell lines, presumably because the farnesylation of these proteins was inadequate for growth or because other GGTase-I substrates are also important for cell growth.

Myeloid cells are viable in the absence of Pggt $1 b$. To further explore the effects of Pggt1b deficiency in mammalian cells, we bred Pggt $1 b_{f l / f l}$ mice with lysozyme M-Cre-transgenic mice (LC mice; mice expressing Cre under the control of a myeloid-specific lysozyme $\mathrm{M}$ promoter [ref. 31]). Pggt1 $b^{f l /+} \mathrm{LC}$ and Pggt $1 b^{f l / f l} \mathrm{LC}$ mice were healthy and fertile, with normal white blood cell counts and normal histology of the spleen, liver, and bone marrow.

To determine the efficiency of Cre-induced inactivation of Pggt1b, we used quantitative PCR analysis of genomic DNA and cDNA. In bone marrow-derived, in vitro-differentiated macrophages from $P g g t 1 b^{f / f l} \mathrm{LC}$ mice, the efficiency of Cre recombination was $90 \%$ (genomic DNA, $90 \% \pm 2.8 \%$; cDNA, $90 \% \pm 3.1 \%, n=2$ ). 
A

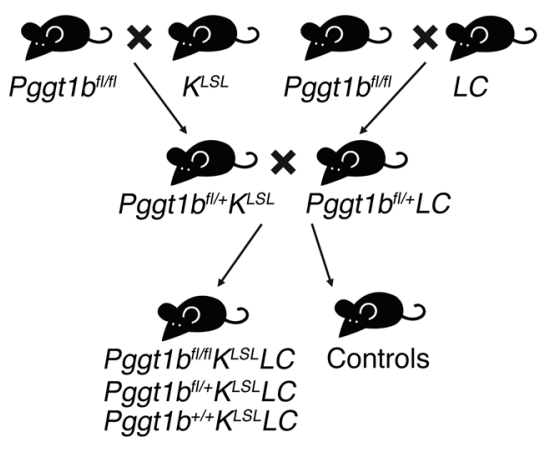

B

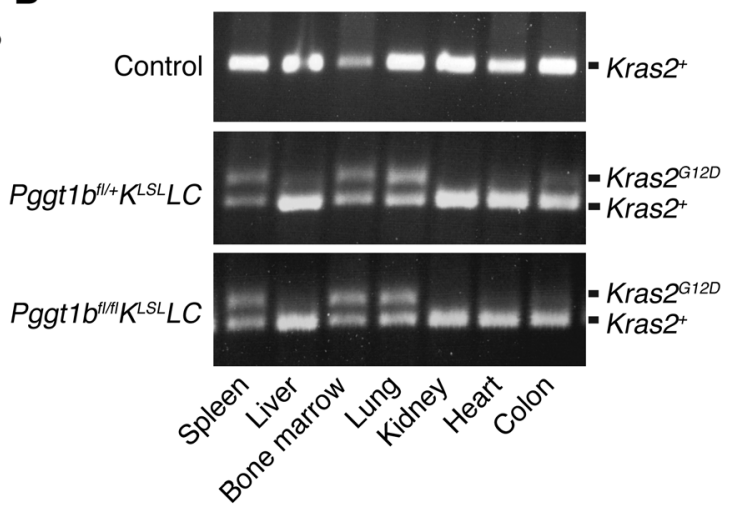

C

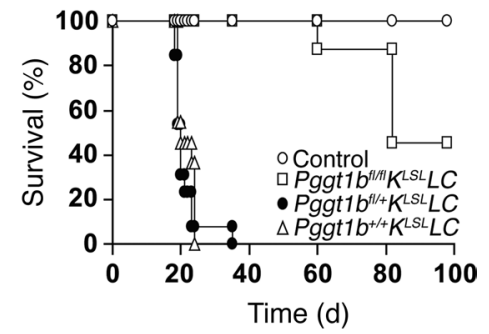

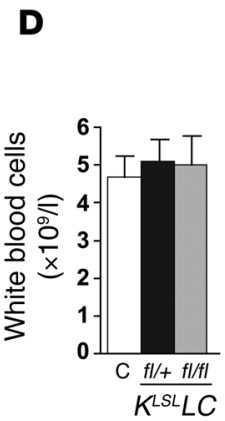

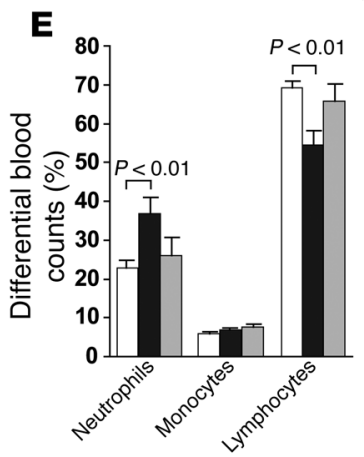

$\mathbf{F}$

G
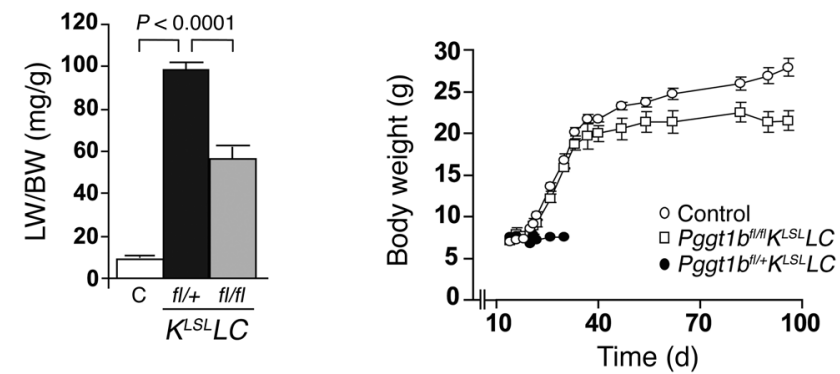

Figure 4

Inactivation of Pggt1b extends the lifespan of mice with LC-induced expression of K-RASG12D. (A) Schematic of the breeding strategy. Control

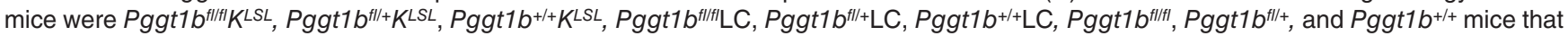
showed no apparent phenotypes. (B) PCR amplification of genomic DNA from tissues to detect the activated $K^{G 12 D}$ allele. (C) Kaplan-Meier curve

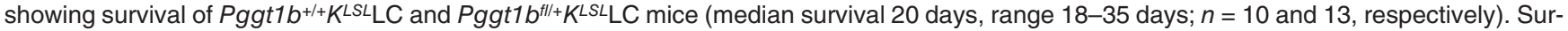
vival was improved in $P g g t 1 b^{f / / f} K^{L L L L C}$ mice (median survival 82 days, range 60 to more than 98 days; $n=9 ; P<0.0001$ versus $P g g t 1 b^{+/+} K^{L S L L C}$ and Pggt $1 b^{f l+} K^{L S L L C ~ m i c e) . ~ A l l ~ c o n t r o l ~ m i c e ~}(n=15)$ were alive at day 98, when the experiment was terminated. (D) Total white blood cell counts in control ( $n=26$; white bar), Pggt $1 b^{f l /}+K^{L S L L C ~}\left(n=18\right.$; black bar), and Pggt $1 b^{f / f / I} K L S L L C$ ( $n=9$; gray bar) mice at 3 weeks of age. Values are mean \pm SEM. (E) Percentage of neutrophils, monocytes, and lymphocytes in the blood from control $\left(n=26\right.$; white bars), $P g g t 1 b^{f l /+} K^{L S L L C}$ ( $n=18$; black bars), and Pggt1 $b^{t / / 1 / 1} K^{L S L L C ~(~} n=9$; gray bars) mice at 3 weeks of age. Values are mean \pm SEM. (F) Lung weight relative to total body weight (LW/BW) in control $\left(n=9\right.$; white bar), Pggt1 $b^{f /+}+K^{L S L L C ~}\left(n=15\right.$; black bar), and Pggt1 $b^{f / l f l} K^{L S L L C}(n=11$; gray bar) mice at 3 weeks of age. Values are mean \pm SEM. $(\mathbf{G})$ Body weight of male controls $\left(n=3-14\right.$, depending on the time point) and $P g g t 1 b^{f l / f l} K^{L S L L C}$ (day $14-89$, $n=3-12$; day 90-96, $n=2$ ), and $P g g t 1 b^{f l+} K^{L S L L C ~(d a y ~ 14-20, ~} n=6-14$; day 21-30, $n=1-2$ ) mice. Values are mean \pm SEM.

In unsorted thioglycollate-elicited peritoneal macrophages, the efficiency was somewhat lower $(77 \%-89 \%)$. The yield of macrophages from different groups of mice did not differ, and more than $90 \%$ of monolayer cells were positive for the macrophage cell surface markers galectin-3 (MAC-2) and sialoadhesin (MAC-3), as judged by immunofluorescence microscopy. In genomic DNA from circulating white blood cells of $P g g t 1 b^{f l / f l} \mathrm{LC}$ mice, the extent of Cre recombination was $20 \% \pm 2.8 \%(n=3)$. The mean percentage of myeloid cells in blood of the same mice was $25 \% \pm 0.5 \%$, suggesting approximately $80 \%$ efficiency of Cre recombination in circulating myeloid cells.

A knockout of Pggt1b ameliorates phenotypes and extends the lifespan of mice expressing $K-R A S^{G 12 D}$. To determine the effect of inactivating Pggt $1 b$ during K-RAS-induced transformation in vivo, we produced mice that were heterozygous for the $K^{L S L}$ allele (30) and the LC allele (31) on a background of the conditional Pggt $1 b$ allele (Figure 4A). Because LC mice have primarily been used in studies of conditional allele inactivation in myeloid cells (32-34), we suspected that $K^{L S L} L C$ mice would develop a K-RAS ${ }^{\text {G12D-induced }}$ myeloid malignancy. However, lysozyme $\mathrm{M}$ is also known to be expressed in type II pneumocytes in the lung $(35,36)$. Indeed, the dominant phenotype of the $K^{L S L} \mathrm{LC}$ mice was lung cancer (described further below).

The $K^{G 12 D}$ allele in $K^{L S L} L C$ mice was activated in bone marrow and spleen, as expected, but it was also activated in lung (Figure 4B). The phenotypes of $P g g t 1 b^{+/+} K^{L S L} \mathrm{LC}$ and $P g g t 1 b^{f l /+} K^{L S L} \mathrm{LC}$ mice were indistinguishable from each other. Both appeared normal until they were 17 days old. At that point, however, their health deteriorated rapidly, and more than $95 \%$ of the mice died or had to be euthanized due to severe dyspnea and reduced body weight (median survival, day 20; maximum survival, day 24 and day 35, respectively) (Figure 4C). Although K-RASG12D was expressed in myeloid cells, white blood cell counts were not increased in the Pggt $1 b^{f l+} K^{L S L}$ LC mice (Figure 4D); however, the percentage of neutrophils in the peripheral blood was higher and the percentage of lymphocytes was lower than in littermate controls lacking the $K^{L S L}$ and LC alleles (Figure 4E). The most striking phenotype of the Pggt $1 b^{f l /}{ }^{L} K^{L S L} L C$ mice was a 10 -fold increase in lung weight at days 18-22 (Figure 4F) that resulted from lung tumors (see below). 

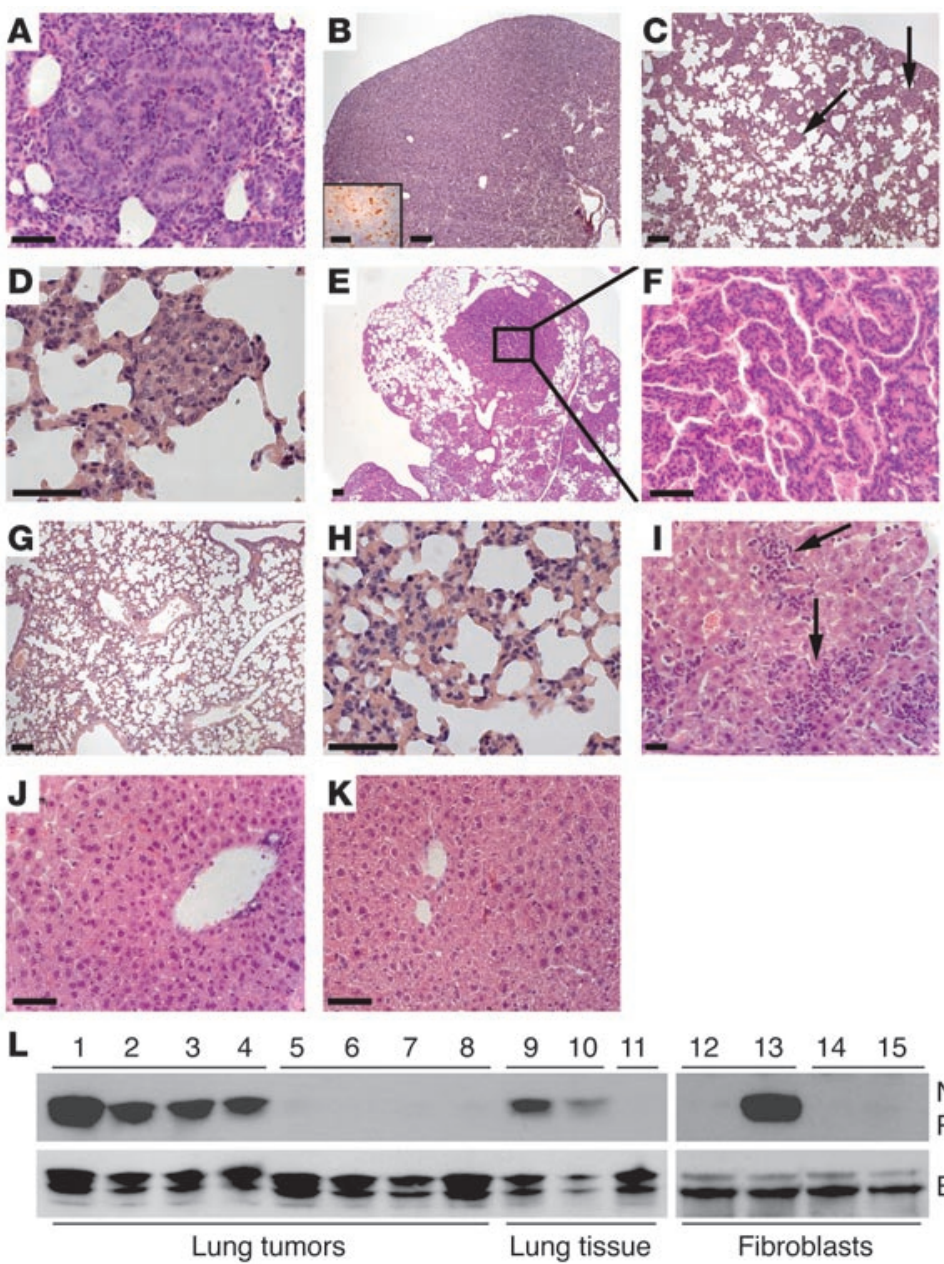

Nonprenylated RAP1

ERK $1 / 2$

Fibroblasts

\section{Figure 5}

Pggt $1 b^{f /+} K^{L S L L C}$ mice develop lung cancer and hepatic leukocyte infiltration, and Pggt1b deficiency ameliorates these phenotypes. (A-K) H\&Estained sections of lung and liver. (A) Advanced adenoma in lung of a day $11 \mathrm{Pggt1} \mathrm{b}^{\mathrm{fl}+} K^{L S L L C}$ mouse. (B) Diffuse adenocarcinoma that obliterates the majority of alveolar spaces in lung of a day $20 \mathrm{Pggt} 1 \mathrm{~b}^{\mathrm{fl}+} \mathrm{K}^{\mathrm{LSLLC}}$ mouse; inset shows $\mathrm{Ki}-67$ immunostaining. (C) AAH lesions (arrows) in lung of a day $20 \mathrm{Pggt} 1 \mathrm{~b}^{\mathrm{ft} / \mathrm{I}} \mathrm{K}$ LSLLC mouse. (D) Magnification of AAH lesion indicated by left arrow in C. (E) Papillary adenoma in lung of a day 98 $P g g t 1 b^{f l / f I} K^{L S L L C}$ mouse. (F) Magnification of $\mathbf{E}$. (G) Normal lung of a day 11 control mouse. (H) Magnification of G. (I) Leukocyte infiltration in liver of a Pggt $1 \mathrm{~b}^{f l+}+K L S L L C$ mouse; arrows indicate clusters of leukocytes. (J) Normal appearance of liver from a day 62 Pggt1 $b^{f / f t} K^{L S L L C}$ mouse. (K) Normal liver from a day 17 control mouse. Scale bars: $50 \mu \mathrm{m}$ (A, inset in B, D, F, H, and I-K); $100 \mu \mathrm{m}$ (B, C, E, and G). (L) Western blot showing high levels of nonprenylated RAP1 in lung tumors from Pggt1 $b^{f / / f l} K^{L S L L C}$ mice (lanes 1-4) and Creadenovirus-treated $P g g t 1 b^{f / / t}$ fibroblasts (lane 13) and lower levels in lung tissue from Pggt $1 b^{f / f t} \mathrm{LC}$ mice (lanes 9 and 10). Nonprenylated RAP1 was undetectable in lung tumors from Pggt $1 b^{f / /} K^{L S L L C}$ mice (lanes 5-8), normal lung tissue of Pggt $1 b^{f /+}+\mathrm{LC}$ mice (lane 11), $\beta$-gal-adenovirus-treated Pggt $1 b^{f / f t}$ (lane 12) and Pggt1 $b^{f /++}$ (lane 14) fibroblasts, and Cre-adenovirus-treated Pggt $1 b^{f / /+}$ fibroblasts (lane 15). Total ERK1/2 expression was analyzed on the same blot as a loading control. Protein extracts from an additional 4 tumors of $P g g t 1 b^{f / l f l} K K^{L S L L C}$ mice and 2 tumors from Pggt $1 b^{f /+} K^{L S L L C}$ mice were analyzed with similar results.
The survival of mice was monitored for 14 weeks; on day 98 , all surviving mice were euthanized. $P g g t 1 b^{f l / f l} K^{L S L} L C$ mice $\left(K^{L S L} L C\right.$ mice that were homozygous for the Pggt $1 b$ conditional allele) survived far longer than $P g g t 1 b^{f l /}+K^{L S L} L C$ mice, some for the entire 98 days (Figure 4C; $P<0.0001$ ). Pggt $1 b^{f l / f l} K^{L S L} L C$ mice gained weight at the same rate as control mice until day 40 and then grew more slowly (Figure 4G). At days 18-22, the percentages of neutrophils and lymphocytes in $P g g t 1 b^{f l / f l} K^{L S L} L C$ mice were entirely normal (Figure 4E), and the white blood cell counts remained low throughout the experiment. The lung weight was lower in Pggt $1 b^{f / f l} K^{L S L}$ LC than in Pggt $1 b^{f l /+} K^{L S L}$ LC mice but was still higher than in control mice (Figure $4 \mathrm{~F}$ ).

Inactivation of Pggt $1 b$ delays the onset and reduces severity of K-RASinduced lung cancer. The Pggt $1 b^{f l /+} K^{L S L} L C$ mice developed lung tumors that ranged from atypical adenomatous hyperplasia (AAH), adenoma, and adenocarcinoma at day 11 (Figure 5A) to a diffuse adenocarcinoma that obliterated alveolar spaces by day 20 (Figure 5B), undoubtedly explaining the dyspnea and premature death. In contrast, the Pggt $1 b^{f l / f l} K^{L S L} \mathrm{LC}$ mice exhibited normal lung histology with only very mild AAH at day 11. At day 20 (Figure 5, $\mathrm{C}$ and D) and day 62, the lungs of $P g g t 1 b^{f l / f l} K^{L S L} \mathrm{LC}$ mice contained a few AAH lesions, but many segments of the lungs retained entirely normal histology. By day 98, the AAH had progressed and scattered adenomas were evident (Figure 5, E and F). Lung histology in the control mice is shown in Figure 5, G and H. Pggt $1 b^{f l /}{ }^{K L S L} \mathrm{LC}$ mice had an infiltration of myeloid cells in the liver (Figure 5I). In contrast, $P g g t 1 b^{f / f l} K^{L S L}$ LC mice had entirely normal livers at days 20 , 62 (Figure 5J), and 98, that were indistinguishable from those of control mice (Figure $5 \mathrm{~K}$ ). The lung tumors of $P g g t 1 b^{f l /}{ }^{+} K^{L S L} L C$ and $P g g t 1 b^{f l / f l} K^{L S L} L C$ mice were negative for the monocyte/macrophage marker CD11b, the Clara cell marker CC10, and the T cell markers CD4 and CD8 but were positive for the type II pneumocyte marker SP-C as judged by immunohistochemistry (Figure 6).

Both alleles of $P g g t 1 b$ are inactivated in lungtumors of Pggt $1 b^{f l / f l} K^{L S L} L C$ mice. To determine whether both alleles of Pggt $1 b$ had been inactivated in the lung tumors of $P_{g g t} 1 b^{f l f f l} K^{L S L} L C$ mice, we performed quantitative PCR and Western blots. The tumor cells expressed K-RAS ${ }^{\mathrm{G} 12 \mathrm{D}}$ and lacked the stop cassette in the promoter of the $K^{L S L}$ allele. By quantitative PCR of genomic DNA ( $n=10$ tumors from 5 mice), the amount of the stop cassette was reduced by $68 \% \pm 1.7 \%$ in the tumors of $P g g t 1 b^{f l / f l} K^{L S L} \mathrm{LC}$ mice, indicating that $68 \%$ of the cells in the tumor were tumor cells. Theoretically, if both alleles of $P g g t 1 b$ had been inactivated in all of the tumor cells, there would be approximately a 68\% reduction in Pggt $1 b$. Indeed, Pggt $1 b$ levels were reduced by $65 \% \pm 1.7 \%$ (the reductions in the stop cassette and Pggt $1 b$ were not different; $P=0.13$ ). In addition, nonprenylated RAP1 was present at high levels in tumor extracts from $P g g t 1 b^{f l / f l} K^{L S L} L C$ mice but was never detected in tumors from $P g g t 1 b^{f l /} K^{L S L} L C$ mice or in Cre-adenovirus-treated Pggt $1 b^{f l+}$ fibroblasts, as shown by Western blots (Figure 5L). Moreover, the levels of nonprenylated RAP1 were 


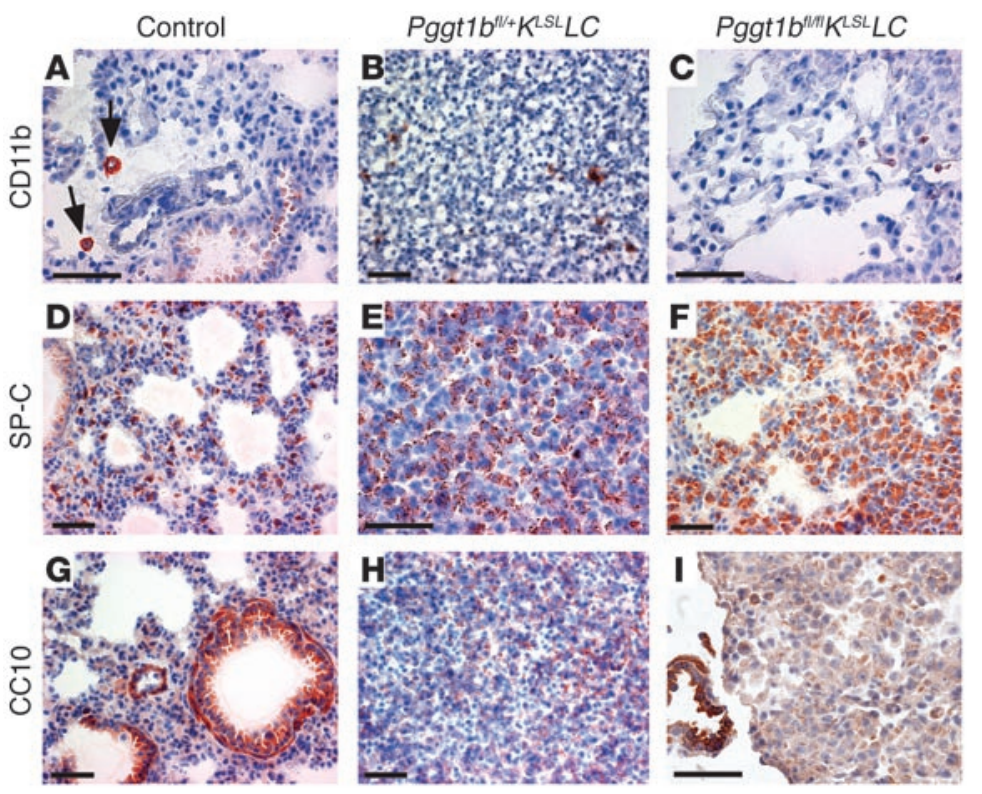

\begin{abstract}
Figure 6
Immunophenotype of lung tumors of day $17 \mathrm{Pggt}^{\mathrm{fll}+} \mathrm{K}^{\mathrm{LSLLC}}$ and Pggt1 $b^{f l / f l} K L S L L C$ mice. (A) Expression of CD11b in macrophages in lung of control mouse (arrows). (B and $\mathbf{C}$ ) CD11b-negative tumors in lungs of $P g g t 1 b^{f l+}+K^{L S L L C ~(B) ~}$ and Pggt $1 b^{f / l t l} K L S L L C$ (C) mice. (D) SP-C staining in lung of control mouse. (E and F) SP-C-positive tumors in lungs of $P g g t 1 b^{f / l+} K^{L S L L C ~(E) ~ a n d ~ P g g t 1 ~} b^{f / / t} K^{L S L L C ~(F) ~ m i c e . ~(G) ~}$ CC10-positive bronchiole in lung of control mouse. (H) CC10negative tumor in lung of $P g g t 1 b^{f l /+} K^{L S L L C}$ mouse. (I) CC10positive bronchiole (lower left) next to CC10-negative lesion in lung of $P g g t 1 b^{f / / f I} K^{L S L L C}$ mouse. Scale bars: $50 \mu \mathrm{m}$.
\end{abstract}

2.4-fold higher in lung tumors of $P_{g g t} 1 b^{f l / f l} K^{L S L} \mathrm{LC}$ mice than in the lungs from $P g g t 1 b^{f l / f l} \mathrm{LC}$ mice, as judged by densitometric analyses of Western blots ( $P<0.01, n=4$; Figure $5 \mathrm{~L}$ and data not shown). These data strongly suggest that lung tumors in $P g g t 1 b^{f l / f l} K^{L S L} L C$ mice arose from cells that accumulate nonprenylated RAP1 (i.e., cells in which both Pggt1 $b$ alleles had been inactivated).

Inactivation of Pggt1b inbibits proliferation and colony growth of $K-R A S^{G 12 D}$-expressing hematopoietic cells. Spleens from Pggt $1 b^{f l+} K^{L S L} \mathrm{LC}$ mice contained an increased proportion of GR-1 $1^{+}, \mathrm{CD} 11 \mathrm{~b}^{+}$, and CD $117^{+}$cells, consistent with expansion of a pool of immature myeloid cells (Figure 7A). We hypothesized that these splenocytes would grow autonomously in vitro. Indeed, splenocytes from Pggt $1 b^{f l /+} K^{L S L}$ LC mice formed colonies in the absence of growth factors, whereas splenocytes from control and $P g g t 1 b^{f l / f l} K^{L S L} L C$ mice did not (Figure 7B). Similar results were obtained with bone marrow cells (Figure 7C). In the presence of growth factors (SCF, IL-3, IL-6, erythropoietin), bone marrow cells from $P g g t 1 b^{f l+}{ }^{L L S L} L C$, $P g g t 1 b^{f l f l} K^{L S L} L C$, and control mice formed similar numbers of colonies (Figure 7D). However, there were more GM-CFUs in the bone marrow of Pggt $1 b^{f l /+} K^{L S L}$ LC mice than in $P g g t 1 b^{f l / f l} K^{L S L} L C$ or control mice (Figure 7D; $P<0.01$ ). Genotyping of the GM-CFU colonies shown in Figure 7D demonstrated that the $K^{L S L}$ allele was activated and that both Pggt1b alleles were inactivated (Figure 7E).

\section{Discussion}

In this study, we created mice with a conditional Pggt $1 b$ knockout allele and show that the inactivation of Pggt $1 b$ arrested proliferation and reduced migration of cultured fibroblasts; it also improved survival and reduced lung tumor formation and myeloproliferation in $K^{L S L} L C$ mice. Moreover, several cell types were viable in the absence of Pggt $1 b$, including fibroblasts, macrophages, lung tumor cells, and cells within GM-CFU colonies.

In normal fibroblasts, inactivation of Pggt $1 b$ arrested proliferation, disrupted the actin cytoskeleton, increased the levels of $\mathrm{P} 21^{\mathrm{CIP} 1}$, and reduced cell migration. In fibroblasts expressing K-RAS ${ }^{\mathrm{G} 12 \mathrm{D}}$, the absence of Pggt $1 b$ did not increase p $21^{\mathrm{CIP} 1}$ levels and resulted in cell rounding. A potential mechanism behind the cytoskeletal disruption and proliferation arrest in Pggt1b-deficient cells is inhibition of geranylgeranylation of the RHO family proteins such as RHOA and CDC42. In keeping with this idea, expressing fRHOA and fCDC42 prevented cell rounding in Pggt1b-deficient K-RAS ${ }^{\mathrm{G} 12 \mathrm{D}}$-expressing fibroblasts. However, the fRHOA and fCDC42 did not fully restore cell proliferation, suggesting that additional GGTase-I substrates are required for normal levels of cell proliferation.

The inactivation of Pggt $1 b$ significantly reduced lung tumor growth and improved survival in $K^{L S L} L C$ mice. However, tumors eventually developed in the Pggt $1 b^{f l / f l} K^{L S L} L C$ mice. Theoretically, these tumors might have arisen from K-RAS ${ }^{\mathrm{G} 12 \mathrm{D}}$-expressing cells in which Pggt $1 b$ was not completely inactivated. In fact, such a scenario was recently proposed to explain the results of experiments designed to assess the impact of Rac1 deficiency on K-RAS-induced lung cancer (37). However, in the current study, we provide strong evidence that both alleles of Pggt1b were inactivated in lung tumor cells expressing K-RAS ${ }^{\mathrm{G} 12 \mathrm{D}}$.

The finding that both alleles of Pggt1b were inactivated in K-RAS ${ }^{\text {G12D }}$-expressing lung tumors suggests that mammalian cells can proliferate in the absence of GGTase-I activity. This notion is further supported by the finding that both Pggt $1 b$ alleles were inactivated in individual GM-CFU colonies from bone marrow of Pggt $1 b^{f l / f l} K^{L S L}$ LC mice. Why would the tumor cells and bone marrow cells be different from the cultured fibroblasts, which never proliferated in the absence of GGTase-I? One possibility is that the tumor cells accumulated additional mutations that allowed them to overcome the effect of Pggt $1 b$ deficiency. Another possibility is that alternate prenylation of key substrates by FTase was somehow more efficient in Pggt1b-deficient tumor cells. Some CAAX proteins, such as RHOB, KRAS, and NRAS, can be prenylated by both FTase and GGTase-I $(38,39)$. Perhaps the tumor cells, but not the fibroblasts, eventually accumulated sufficient amounts of farnesylated proteins to permit cell growth.

In addition to lung cancer, activation of the $K^{L S L}$ allele by the LC allele resulted in a myeloproliferative phenotype, with infiltration of leukocytes in the liver and growth factor-independent colony growth of hematopoietic cells. Both of those phenotypes were eliminated in the absence of GGTase-I in $P g g t 1 b^{f / f l} K^{L S L} L C$ mice, and the white blood cell counts in the Pggt $1 b^{f l / f l} K^{L S L} L C$ mice remained 
A

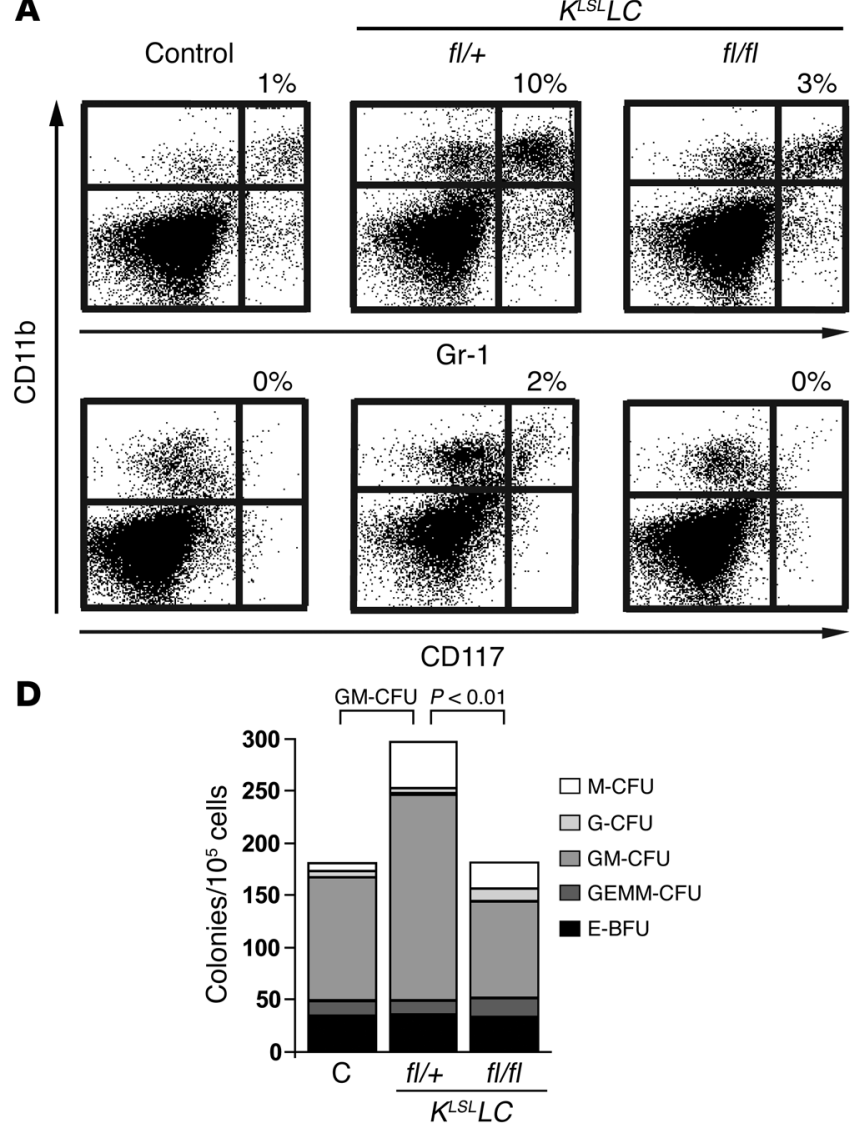

B
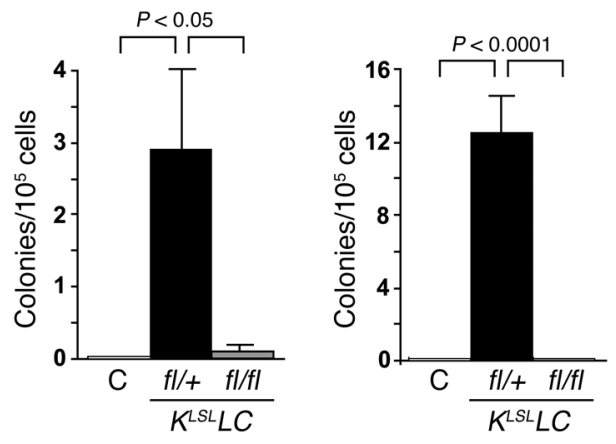

E

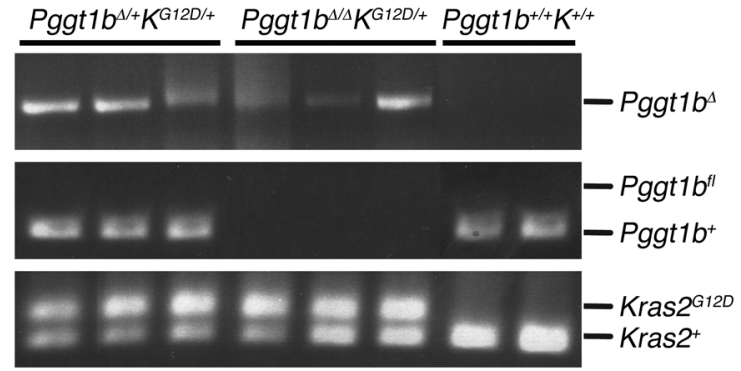

Figure 7

Pggt1b deficiency eliminates K-RAS-induced myeloproliferation. (A) Flow cytometry showing an increased percentage of CD11b/Gr-1 (upper panels) and CD11b/CD117 (lower panels) double-positive cells in the spleens of Pggt $1 b^{f /+} K^{L S L L C}$ mice compared with control and Pggt1 $b^{f l / f I} K^{L S L L C}$ mice $(n=3$ mice of each genotype). Shown are representative scatter plots of data from 1 mouse of each genotype and the mean percentage of double-positive cells. The increase in double-positive cells in spleens of $P g g t 1 b^{f /+}+K^{L S L L C}$ mice was statistically significant $(P<0.001$ versus control and Pggt1 $b^{f / f f l} K L S L L C$ mice). (B) Growth factor-independent colony growth of splenocytes from control $(n=4), P g g t 1 b^{f / l+} K L S L L C(n=5)$, and Pggt1 $b^{f l / f I} K$ LSLLC $(n=5)$ mice. Splenocytes were seeded in methylcellulose medium, and the colonies were counted after 10 days. Values are mean \pm SEM. (C) Growth factor-independent colony growth of bone marrow cells from control $(n=4), P g g t 1 b^{f l+}+K^{L S L L C ~}(n=6)$, and $P g g t 1 b^{f l / t} K K^{L S L}$ LC $(n=5)$ mice. Bone marrow cells were seeded in methylcellulose medium, and colonies were counted after 10 days. Values are mean \pm SEM. (D) Colony growth of bone marrow cells from control, Pggt $1 b^{f / /}+K K^{L S L L C}$, and Pggt1 $b^{f / f t} K^{L S L L C}$ mice $(n=2$ of each genotype) in the presence of growth factors. Cells were seeded in methylcellulose medium supplemented with recombinant SCF, IL-3, IL-6, and erythropoietin, and the colonies were counted and morphologically typed 10 days later. E-BFU, burst-forming unit-erythroid; GEMM, granulocyte, erythrocyte, macrophage, megakaryocyte. (E) PCR amplification of genomic DNA from individual GM-CFU bone marrow colonies from the experiment in D.

low for the entire length of their lifespan (up to 98 days). In the future, it would be interesting to define the impact of GGTase-I deficiency on other experimental myeloproliferative disease syndromes, for example the one elicited when the $K^{L S L}$ allele is activated by the Mx1-Cre transgene $(40,41)$.

In a recent study, inactivating the $\beta$ subunit of FTase (Fntb) did not alter the development of K-RAS-induced lung cancer and had only a minor effect on H-RAS-induced skin cancer (42). Also, Fntbdeficient fibroblasts grew in cell culture, albeit at a reduced rate. The current study indicates that GGTase-I deficiency has a greater impact than FTase deficiency on cell proliferation, migration, and K-RAS-induced transformation. Multiple factors likely contribute to this difference. One is that there are simply more geranylgeranylated than farnesylated proteins in cells (1). It is also possible that the lipid modification is more important for geranylgeranylated proteins. A geranylgeranyl lipid is significantly more hydro- phobic than a farnesyl lipid (43), so it seems plausible that the loss of protein geranylgeranylation could have a greater effect on the avidity of proteins for membrane surfaces, and hence greater functional consequences, than loss of protein farnesylation.

The conditional Pggt1b allele we developed will make it feasible to compare the impact of genetic and pharmacologic inhibition of GGTase-I. Such studies could be enlightening because they might help to sort out mechanism-related versus compoundrelated effects of different GGTIs. Previously, GGTIs were reported to upregulate $\mathrm{p} 21^{\mathrm{CIP} 1}$ and induce apoptosis in cultured cells $(27,28,44,45)$. However, in the current study, p21 ${ }^{\mathrm{CIP} 1}$ levels were not increased in K-RAS ${ }^{\mathrm{G} 12 \mathrm{D}}$-expressing Pggt1b-deficient cells. The explanation for this difference is not known. However, now that a genetic model of GGTase-I deficiency is in hand, it should be possible to define which effects of GGTIs are due to inhibition of GGTase-I and which are likely to be due to off-target effects of the GGTI. 


\section{Methods}

Generation of a conditional Pggt1b knockout allele. A 765-bp fragment containing exon 7 and parts of flanking introns from Pggt $1 b$ was amplified with primers $5^{\prime}$-GTTCTGTTTTTCCAGTTGGCACGGG-3' and 5'-GGTCATAGTGACTCTTCATAGCAGTGAAACCC-3' using genomic DNA from $129 /$ OlaHsd ES cells as a template. The fragment was cloned into the EcoRI site of pNB1, which contains a polylinker flanked by loxP sites. The floxed exon 7 fragment was cloned into the SalI site of pKSloxPNTmod, a commonly used plasmid for gene-targeting constructs (46). In this vector, the loxP sites flanking the neo cassette had been replaced with Frt sites. A 2.4-kb $5^{\prime}$ arm was amplified with primers 5'-AGCGATATCTTTGGAGTAGAGTGTATTCTAGGTTGGGG-3' and 5'-AGCAGCGATATCCTTACACCAGAACTAACCAACCCCC- $3^{\prime}$ and cloned into the BamHI site of the targeting vector. Finally, a 5.3-kb 3' arm was amplified with primers 5'-AGCAGCGGCGCGCCCCATTATCATCACGGTGGGAAGCATGGCAGTG-3' and 5'-GTCGTCTTAATTAAGCTAAGAGAGCACTGGTTGCTCTGAGTTGCAGTATCC-3' and cloned into the PacI and AscI sites of the vector. The vector was linearized with $\mathrm{XhoI}$ and electroporated into 129/OlaHsd ES cells. After selection in G418 for 10 days, 306 colonies were screened by Southern blotting of SpeI-digested genomic DNA with a 3 '-flanking probe. Two targeted clones (Pggt $\left.1 b^{f /+}\right)$ were used to generate chimeric mice, which transmitted the mutation to their offspring.

Mouse breeding. Pggt $1 b^{\text {fl/fl}}$ mice were bred with mice that were heterozygous for a Cre-inducible $K^{L S L}$ allele (30), generating Pggt $1 b^{f / /+} K^{L S L /+}$ mice. The $K^{L S L}$ allele contains an activating mutation (G12D) and a floxed transcriptional terminator sequence. Pggt $1 b^{f / f l}$ mice were also bred with LC mice (The Jackson Laboratory) to produce Pggt $1 b^{f / /}+\mathrm{LysM}-\mathrm{Cre}^{+/ 0}$ mice (designated Pggt $1 b^{f / /+} \mathrm{LC}$ ). The LC allele (31) yields expression of Cre recombinase in granulocytes, monocytes, macrophages, and type II pneumocytes. Finally, we bred Pggt $1 b^{f l+} \mathrm{K}^{L S L}$ mice with Pggt $1 b^{f /+} \mathrm{LC}$ mice to produce $P g g t 1 b^{+/+} K^{L S L} \mathrm{LC}$, Pggt $1 b^{f l /+} K^{L S L} \mathrm{LC}$, and Pggt $1 b^{f / f l} K^{L S L} \mathrm{LC}$ mice, in which Cre simultaneously activates the expression of K-RAS ${ }^{\mathrm{G} 12 \mathrm{D}}$ and inactivates the expression of the $P g g t 1 b^{f l}$ allele. Control mice inherited either the $K^{L S L}$ or LC allele, but not both. The mice had a mixed genetic background (129/SV and C57BL/6). Mouse experiments were approved by the Animal Research Ethics Committee at Göteborg University.

Genotyping. The Pggt $1 b^{f l}$ allele was genotyped by PCR amplification of genomic DNA from tail biopsies with forward primer 5'-CCTGAATGCAGATCTGTGGA-3' and reverse primer $5^{\prime}$-CCTATGAAAGCAGCACGACA-3'. The Pggt $1 b^{+}$and Pggt $1 b^{f l}$ alleles yielded 280 - and 360 -bp products, respectively. The LC allele was genotyped according to the distributor's instructions (http://jaxmice.jax.org/pub-cgi/protocols/protocols.sh?objt ype=protocol\&protocol_id=747); the $K^{L S L}$ and activated $K^{G 12 D}$ alleles were genotyped as described $(30,40)$.

Isolation of embryonic fibroblasts and Cre-adenovirus infection. Experiments with primary fibroblasts (isolated from Pggt $1 b^{f / f l}$, Pggt $1 b^{f / /+}$, Pggt $1 b^{f / f l} K^{L S L}$, and $P g g t 1 b^{f /+} K^{L S L}$ embryos on day 15.5 post coitum) were performed with passage 2-4 cells. To inactivate Pggt $1 b$ and activate K-RAS ${ }^{\mathrm{G} 12 \mathrm{D}}$ expression, $10^{6}$ cells were seeded in $100-\mathrm{mm}$ dishes and infected with $5 \times 10^{7} \mathrm{pfu} / \mathrm{ml}$ of Cre-adenovirus (AdRSVCre; University of Iowa, Iowa City, Iowa, USA) for 24 hours in $5 \mathrm{ml}$ of medium. As a control, the same cell lines were treated with an adenovirus encoding $\beta$-gal (AdRSVnlac $Z$; University of Iowa).

Determining the efficiency of Cre recombination. DNA and total RNA were prepared from fibroblasts, macrophages, tissues, and blood from mice. cDNA was synthesized from equal amounts of total RNA with the iScript cDNA Synthesis Kit (Bio-Rad). Genomic DNA and cDNA were used for real-time quantitative PCR with Power SYBR Green PCR Master Mix on an ABI Prism 7900HT Sequence Detection System (Applied Biosystems). The following primer pairs were used: Pggt1b genomic DNA, 5'-CATGTCGTGCTGCTTTCATA-3' and 5'-CAGTTTACCCATCAGGCACA-3'; STOP cas- sette in $K^{L S L}$ allele genomic DNA, 5'-TCGAGGGACCTAATAACTTCGT-3' and $5^{\prime}$-CATAGTACGCTATACCCTGTGGA-3'; Rce1 genomic DNA (used as a control), 5'-CGAGTAAATCTGTGGGAGAGG-3' and $5^{\prime}$-CGGTGCAATAACTTGGTTTC-3'; Pggt $1 b$ cDNA, 5'-GGGAGCAGGCCTTGAGTCT-3' and 5'-GTTTACCCATCAGGCACAGTGA-3'; and cyclophilin cDNA (used as reference gene), 5'-TGGAGAGCACCAAGACAGACA-3' and 5'-TGCCGGAGTCGACAATGAT-3'. Cre recombination was also assessed by PCR of the Pggt $1 b^{f l}$ allele (described above), the recombined Pggt $1 b^{\Delta}$ allele (genomic DNA, 5'-CCTGAATGCAGATCTGTGGA-3' and $5^{\prime}$-CCTGATCGACAAGACCGGCTTCCA-3'; product size, approximately 1,300 bp), exon 7-9 sequences (cDNA, 5'-CCTTCTGTGGCATTGCGTCA-3' and 5'-AGCCCATGCTGAAGTATTAG-3'; product size, $600 \mathrm{bp}$ ), and exon 4-9 sequences (cDNA, 5'-AGATGACTTAGGCCGTGTGG-3' and 5'-CGCAGATCCCGAAGTACGC-3'; product sizes, 575 bp [Pggt1 bf] and 390 bp [Pggt 1 $\left.b^{\Delta}\right]$ ).

Prenyltransferase activity measurements. To measure GGTase-I activity, cytosolic preparations from total cellular extracts were incubated with ${ }^{3} \mathrm{H}-$ labeled geranylgeranylpyrophosphate and recombinant human $\mathrm{H}$-RasCys-Val-Leu-Leu; for FTase activity, the cytosolic preparations were incubated with ${ }^{3} \mathrm{H}$-labeled farnesylpyrophosphate and recombinant human H-Ras-Cys-Val-Leu-Ser. These assays have been described (47).

Subcellular fractionation and Western blotting. Soluble and membrane fractions of cultured fibroblasts were prepared by ultracentrifugation at $100,000 \mathrm{~g}$ as described in ref. 48. For Western blotting, equal amounts of total protein from subcellular fractions or total protein extracts from fibroblasts and tissues were size fractionated on 10\%-20\% SDS-PAGE (Criterion; Bio-Rad). The proteins were transferred to nitrocellulose membranes and incubated with antibodies recognizing nonprenylated RAP1A (catalog no. sc-1482), total RAP1 (catalog no. sc-65), RHOA (catalog no. sc-418), p21 CIP1 (catalog no. sc-6246), cyclin D1 (catalog no. sc-450), and the HA tag (catalog no. sc-7392) (Santa Cruz Biotechnology Inc.), phosphorylated ERK1/2

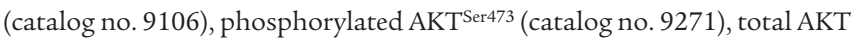
(catalog no. 9272), total ERK1/2 (catalog no. 9102), CDC42 (catalog no. 2462) (Cell Signaling Technology), and the Myc tag (catalog no. R950-25; Invitrogen). Protein bands were visualized with a horseradish peroxidaseconjugated secondary antibody (catalog nos. sc-2314, sc-2313, and sc-2354 [Santa Cruz Biotechnology Inc.] and NA931 [Amersham]) and the ECL Western Blotting System (Amersham Biosciences). Protein bands were analyzed by densitometry with Quantity One 4.4.0 software (Bio-Rad), and the data were normalized to total ERK1/2.

Cell proliferation. Cells $(n=20,000)$ infected with the Cre- or $\beta$-gal-adenovirus were seeded in duplicate 12 -well plates and incubated in serum-free medium overnight. The medium was then replaced with normal medium $(10 \%$ serum), and the cells were trypsinized and counted at defined intervals.

Staining of the actin cytoskeleton. Fibroblasts on chamber slides were fixed in $4 \%$ paraformaldehyde for 15 minutes and permeabilized with $0.1 \%$ Triton $\mathrm{X}-100$ for 5 minutes at room temperature. F-actin was stained with Alexa Fluor 546-labeled phalloidin (Invitrogen) for 20 minutes at room temperature, and nuclei were stained with DAPI. The slides were photographed with a Zeiss Axiocam MRm digital camera mounted on a Zeiss Axioplan 2 fluorescence microscope.

In vitro cell migration assay. To assess cellular migration, Pggt $1 b^{f / f l}$ fibroblasts were infected with Cre- or $\beta$-gal-adenovirus for 72 hours. Then $2 \times 10^{6}$ cells were seeded to confluence in $60-\mathrm{mm}$ dishes and allowed to adhere for 24 hours. The cells were washed with PBS, and an approximately 1-mmwide gap was created by scraping the monolayer with a pipette tip. Photographs were taken at $0,8,24$, and 48 hours, and cells that had migrated into the gap were counted.

Vectors expressing fRHOA and fCDC42. A Myc-tagged fRHOA construct (RHOA-CVLS) was generated by PCR amplification of mouse fibroblast cDNA with forward primer 5'-AGGATCCGAAATGGAACAAAAACTCATCT- 
CAGAAGAGGATCTGATGGCTGCCATCAGGAAGAAACTGG-3' and reverse primer 5'-AGGATCCTCATGACAACACGCACCCAGACTTTTTCTTCCC$3^{\prime}$. A HA-tagged fCDC42 construct (CDC42-CVLS) was generated by PCR amplification of cDNA with forward primer 5'-AGGATCCGAAATGGAATACCCATACGATGTTCCAGATTACGCTATGCAGACAATTAAGTGTGTTGTTGTTGG-3' and reverse primer 5'-AGGATCCTCATGACA GCACACACCTGCGGCTC- $3^{\prime}$. The fragments were cloned into the double-expression vector pVITRO2-mcs (InvivoGen). Fibroblasts were transfected with $3 \mu \mathrm{g}$ of the vector with FuGENE 6 reagent (Roche Diagnostics) before experiments.

Isolation of bone marrow-derived and peritoneal macrophages. Bone marrow cells were plated in conditioned DMEM from CMG14-12 cells (49), which overexpress mouse macrophage CSF. After 7-9 days, differentiated macrophages were harvested for preparation of DNA and total RNA. Peritoneal macrophages were collected 3 days after intraperitoneal injection of $2 \mathrm{ml}$ of thioglycollate (3\%) and cultured in RPMI-1640 medium. After 2-3 days, genomic DNA and total RNA were prepared.

Blood counts. Complete blood counts were measured with a Hemavet 950FS cell counter (Drew Scientific).

Histology and immunohistochemistry. For routine histology, sections $(4 \mu \mathrm{m})$ of paraformaldehyde-fixed tissues were stained with H\&E. For immunohistochemistry, tissue pieces were embedded in Tissue-Tek (Miles Laboratory) and frozen at $-80^{\circ} \mathrm{C}$. Cryosections $(5 \mu \mathrm{m})$ were incubated with antibodies recognizing CD11b (catalog no. 557394), CD4 (catalog no. 553727), CD8 (catalog no. 558733) (BD Biosciences - Pharmingen), Ki-67 (catalog no. M-7249; DakoCytomation), SP-C (catalog no. sc-7705; Santa Cruz Biotechnology Inc.), and CC10 (07-623; Millipore) overnight at $4{ }^{\circ} \mathrm{C}$. The slides were incubated with a biotin-labeled secondary antibody (Vector Laboratories) for 1 hour and then with StreptABComplex (DakoCytomation) and 3-amino-9-ethyl carbazole buffer containing $\mathrm{H}_{2} \mathrm{O}_{2}$. Sections were counterstained with Mayer's hematoxylin. Peritoneal macrophages cultured on chamber slides were incubated with PE-conjugated antibodies recognizing MAC-2 (Cedarlane Laboratories Ltd.) or MAC-3 (BD Biosciences - Pharmingen). The MAC-2 slides were then incubated with a biotin-labeled secondary antibody (Vector Laboratories), followed by incubation with FITC-conjugated streptavidin. DAPI was used to stain nuclei. Slides were photographed with an Axiocam HRc digital camera on a Zeiss Axiophot microscope.

FACS and apoptosis detection. Spleen cells were incubated with antibodies recognizing the cell-surface markers CD3 (catalog no. 553066), CD11b (catalog no. 550993), CD13 (catalog no. 558745), CD14 (catalog no. 553739), CD19 (catalog no. 552854), CD34 (catalog no. 553733), CD45 (catalog no. 557659), CD117 (catalog no. 553355), and Gr-1 (catalog no. 553129) (BD Biosciences - Pharmingen) and analyzed in a FACSAria Flow Cytometer (BD Biosciences). Data were analyzed with FACSDiva software (version 5.0.1; BD
Biosciences). Cell-cycle distribution of fibroblasts was determined by staining with propidium iodide and by flow cytometry. Apoptosis was detected with the Annexin V:FITC Apoptosis Detection Kit I (catalog no. 556547; BD Biosciences) and by RT-PCR to determine the relative levels of BAX and BCL-2 in a semiquantitative fashion (APO-PCR; Sigma-Aldrich).

Methylcellulose colony assays. Spleen cells $\left(10^{5}\right)$ and bone marrow cells $\left(2 \times 10^{4}\right)$ from control, $P g g t 1 b^{f /+} K^{L S L} \mathrm{LC}$, and $P g g t 1 b^{f / f l} K^{L S L} \mathrm{LC}$ mice were seeded in duplicate $30-\mathrm{mm}$ dishes in methylcellulose medium in the absence of growth factors (MethoCult M3234; StemCell Technologies) or in the presence of recombinant SCF, IL-3, IL-6, and erythropoietin (MethoCult M3232; StemCell Technologies). Ten days later, the number and type of colonies were assessed with an inverted microscope.

Statistics. Data are plotted as mean \pm SEM. Differences in cellular migration, concentrations and percentages of white blood cells, lung weights, densitometry of protein bands on Western blots, colony-forming ability of hematopoietic cells, and FACS analyses were determined with 2-tailed Student's $t$ test or 1-way ANOVA with Tukey's procedure. Survival was assessed with the log-rank test.

\section{Acknowledgments}

This study was supported by grants from the Swedish Cancer Society, the Swedish Medical Research Council, the Swedish Children's Cancer Foundation, the Inga-Britt and Arne Lundberg's Fund, and by a grant from the Västra Götalandsregionen (to M.O. Bergo); and by NIH grants GM46372 (to P.J. Casey) and CA103999, CA099506, and AR050200 (to S.G. Young). M.O. Bergo is a research fellow of the Royal Swedish Academy of Sciences. We thank Tyler Jacks for the $K^{L S L}$ mice, Pernilla Jirholt and Maria Heyden for assistance with macrophage preparations and immunofluorescence microscopy, Hans Nordlinder and Aziz Hussein for help with lung histopathology, Stephen Ordway and Rosie Perkins for editorial assistance, and Jessica Jarjoura for assistance with gene-targeting experiments.

Received for publication November 7, 2006, and accepted in revised form January 30, 2007.

Address correspondence to: Martin O. Bergo, Wallenberg Laboratory, Bruna Straket 16, 3rd floor, Sahlgrenska University Hospital, S-413 45 Göteborg, Sweden. Phone: 46-31-342-78-58; Fax: 46-31-82-37-62; E-mail: Martin.Bergo@wlab.gu.se.

Anna-Karin M. Sjogren and Karin M.E. Andersson contributed equally to this work.
1. Reid, T.S., Terry, K.L., Casey, P.J., and Beese, L.S 2004. Crystallographic analysis of CaaX prenyltransferases complexed with substrates defines rules of protein substrate selectivity. J. Mol. Biol. 343:417-433.

2. Casey, P.J., and Seabra, M.C. 1996. Protein prenyltransferases. J. Biol. Chem. 271:5289-5292.

3. Yokoyama, K., Goodwin, G.W., Ghomashchi, F., Glomset, J.A., and Gelb, M.H. 1991. A protein geranylgeranyltransferase from bovine brain: implications for protein prenylation specificity. Proc. Natl. Acad. Sci. U. S. A. 88:5302-5306.

4. Therrien, M., et al. 1995. KSR, a novel protein kinase required for RAS signal transduction. Cell. 83:879-888.

5. Ohya, Y., et al. 1991. Yeast CAL1 is a structural and functional homologue to the DPR1 (RAM) gene involved in ras processing. J. Biol. Chem 266:12356-12360.

6. Ohya, Y., Qadota, H., Anraku, Y., Pringle, J.R., and Botstein, D. 1993. Suppression of yeast geranyl- geranyl transferase I defect by alternative prenylation of two target GTPases, Rho1p and Cdc42p. Mol. Biol. Cell. 4:1017-1025.

7. Kelly, R., et al. 2000. Geranylgeranyltransferase I of Candida albicans: null mutants or enzyme inhibitors produce unexpected phenotypes. J. Bacteriol. 182:704-713.

8. Running, M.P., et al. 2004. Enlarged meristems and delayed growth in plp mutants result from lack of CaaX prenyltransferases. Proc. Natl. Acad. Sci. U. S. A. 101:7815-7820.

9. Casey, P.J., Solski, P.A., Der, C.J., and Buss, J.E. 1989. p21ras is modified by a farnesyl isoprenoid. Proc. Natl. Acad. Sci. U. S. A. 86:8323-8327.

10. Kato, K., et al. 1992. Isoprenoid addition to Ras protein is the critical modification for its membrane association and transforming activity. Proc. Natl. Acad. Sci. U. S. A. 89:6403-6407.

11. Kohl, N.E., et al. 1995. Inhibition of farnesyltransferase induces regression of mammary and salivary carcinomas in ras transgenic mice. Nat. Med.
1:792-797.

12. Basso, A.D., Kirschmeier, P., and Bishop, W.R. 2006. Lipid posttranslational modifications. Farnesyl transferase inhibitors. J. Lipid Res. 47:15-31.

13. James, G., Goldstein, J.L., and Brown, M.S. 1996. Resistance of K-RasB ${ }^{\mathrm{V} 12}$ proteins to farnesyltransferase inhibitors in Rat1 cells. Proc. Natl. Acad. Sci.U.S. A. 93:4454-4458.

14. Olson, M.F., Paterson, H.F., and Marshall, C.J. 1998. Signals from Ras and Rho GTPases interact to regulate expression of $\mathrm{p}^{2} 1^{\text {Waf1/Cip } 1}$. Nature. 394:295-299.

15. Clark, E.A., Golub, T.R., Lander, E.S., and Hynes, R.O. 2000. Genomic analysis of metastasis reveals an essential role for RhoC. Nature. 406:532-535.

16. Lim, K.H., et al. 2005. Activation of RalA is critical for Ras-induced tumorigenesis of human cells. Cancer Cell. 7:533-545.

17. Peterson, Y.K., Kelly, P., Weinbaum, C.A., and Casey, P.J. 2006. A novel protein geranylgeranyltransferase-I inhibitor with high potency, selectivity, and 
cellular activity. J. Biol. Chem. 281:12445-12450.

18. Sun, J., Qian, Y., Hamilton, A.D., and Sebti, S.M. 1998. Both farnesyltransferase and geranylgeranyltransferase I inhibitors are required for inhibition of oncogenic K-Ras prenylation but each alone is sufficient to suppress human tumor growth in nude mouse xenografts. Oncogene. 16:1467-1473.

19. El Oualid, F., Cohen, L.H., van der Marel, G.A., and Overhand, M. 2006. Inhibitors of protein: geranylgeranyl transferases. Curr. Med. Chem. 13:2385-2427.

20. Gelb, M.H., et al. 2006. Therapeutic intervention based on protein prenylation and associated modifications. Nat. Chem. Biol. 2:518-528.

21. Walters, C.E., et al. 2002. Inhibition of Rho GTPases with protein prenyltransferase inhibitors prevents leukocyte recruitment to the central nervous system and attenuates clinical signs of disease in an animal model of multiple sclerosis. J. Immunol. 168:4087-4094.

22. Ye, J., et al. 2003. Disruption of hepatitis C virus RNA replication through inhibition of host protein geranylgeranylation. Proc. Natl. Acad. Sci. U. S. A. 100:15865-15870.

23. Murthi, K.K., et al. 2003. Antifungal activity of a Candida albicans GGTase I inhibitor-alanine conjugate. Inhibition of Rho1p prenylation in C. albicans. Bioorg. Med. Chem. Lett. 13:1935-1937.

24. Lobell, R.B., et al. 2001. Evaluation of farnesyl: protein transferase and geranylgeranyl:protein transferase inhibitor combinations in preclinical models. Cancer Res. 61:8758-8768.

25. Li, X., et al. 2002. Inhibition of protein geranylgeranylation and RhoA/RhoA kinase pathway induces apoptosis in human endothelial cells. J. Biol. Chem. 277:15309-15316.

26. Dan, H.C., et al. 2004. Phosphatidylinositol-3$\mathrm{OH}$ kinase/AKT and survivin pathways as critical targets for geranylgeranyltransferase I inhibitorinduced apoptosis. Oncogene. 23:706-715.

27. Sun, J., et al. 2003. Geranylgeranyltransferase I inhibitor GGTI-2154 induces breast carcinoma apoptosis and tumor regression in H-Ras transgenic mice. Cancer Res. 63:8922-8929.
28. Vogt, A., Sun, J., Qian, Y., Hamilton, A.D., and Sebti, S.M. 1997. The geranylgeranyltransferase-I inhibitor GGTI-298 arrests human tumor cells in G0/G1 and induces $\mathrm{p} 21$ (WAF1/CIP1/SDI) in a p53-independent manner. J. Biol. Chem. 272:27224-27229.

29. Zhang, F.L., Moomaw,J.F., and Casey, P.J. 1994. Properties and kinetic mechanism of recombinant mammalian protein geranylgeranyltransferase type I. J. Biol. Chem. 269:23465-23470.

30. Jackson, E.L., et al. 2001. Analysis of lung tumor initiation and progression using conditional expression of oncogenic K-ras. Genes Dev. 15:3243-3248.

31. Clausen, B.E., Burkhardt, C., Reith, W., Renkawitz, R., and Förster, I. 1999. Conditional gene targeting in macrophages and granulocytes using LysMcre mice. Transgenic Res. 8:265-277.

32. Rupec, R.A., et al. 2005. Stroma-mediated dysregulation of myelopoiesis in mice lacking I kappa B alpha. Immunity. 22:479-491.

33. Kimura, A., et al. 2004. SOCS3 is a physiological negative regulator for granulopoiesis and granulocyte colony-stimulating factor receptor signaling. J. Biol. Chem. 279:6905-6910.

34. Herbert, D.R., et al. 2004. Alternative macrophage activation is essential for survival during schistosomiasis and downmodulates $\mathrm{T}$ helper 1 responses and immunopathology. Immunity. 20:623-635.

35. Singh, G., Katyal, S.L., Brown, W.E., Collins, D.L., and Mason, R.J. 1988. Pulmonary lysozyme-a secretory protein of type II pneumocytes in the rat. Am. Rev. Respir. Dis. 138:1261-1267.

36. Markart, P., et al. 2004. Comparison of the microbicidal and muramidase activities of mouse lysozyme $\mathrm{M}$ and P. Biochem. J. 380:385-392.

37. Kim, C.F., et al. 2005. Mouse models of human non-small-cell lung cancer: raising the bar. Cold Spring Harb. Symp. Quant. Biol. 70:241-250.

38. Adamson, P., Marshall, C.J., Hall, A., and Tilbrook, P.A. 1992. Post-translational modifications of p21rho proteins. J. Biol. Chem. 267:20033-20038.

39. Whyte, D.B., et al. 1997. K- and N-Ras are geranylgeranylated in cells treated with farnesyl protein transfer- ase inhibitors. J. Biol. Chem. 272:14459-14464.

40. Braun, B.S., et al. 2004. Somatic activation of oncogenic Kras in hematopoietic cells initiates a rapidly fatal myeloproliferative disorder. Proc. Natl. Acad. Sci. U. S. A. 101:597-602

41. Chan, I.T., et al. 2004. Conditional expression of oncogenic $K$-ras from its endogenous promoter induces a myeloproliferative disease. J. Clin. Invest. 113:528-538. doi:10.1172/JCI200420476.

42. Mijimolle, N., et al. 2005. Protein farnesyltransferase in embryogenesis, adult homeostasis, and tumor development. Cancer Cell. 7:313-324.

43. Silvius, J.R., and l'Heureux, F. 1994. Fluorimetric evaluation of the affinities of isoprenylated peptides for lipid bilayers. Biochemistry. 33:3014-3022.

44. Adnane, J., Bizouarn, F.A., Qian, Y., Hamilton, A.D., and Sebti, S.M. 1998. p21(WAF1/CIP1) is upregulated by the geranylgeranyltransferase I inhibitor GGTI-298 through a transforming growth factor beta- and Sp1-responsive element: involvement of the small GTPase rhoA. Mol. Cell. Biol. 18:6962-6970.

45. Miquel, K., et al. 1997. GGTI-298 induces G0-G1 block and apoptosis whereas FTI-277 causes G2-M enrichment in A549 cells. Cancer Res. 57:1846-1850.

46. Hanks, M., Wurst, W., Anson-Cartwright, L., Auerbach, A.B., and Joyner, A.L. 1995. Rescue of the En-1 mutant phenotype by replacement of En-1 with En-2. Science. 269:679-682.

47. Thissen, J.A., and Casey, P.J. 1996. Kinetics of protein farnesyltransferase: sigmoidal vs hyperbolic behavior as a function of assay conditions. Anal. Biochem. 243:80-85.

48. Bergo, M.O., et al. 2004. Inactivation of Icmt inhibits transformation by oncogenic K-Ras and B-Raf. J. Clin. Invest. 113:539-550. doi:10.1172/ JCI200418829.

49. Takeshita, S., Kaji, K., and Kudo, A. 2000. Identification and characterization of the new osteoclast progenitor with macrophage phenotypes being able to differentiate into mature osteoclasts. J. Bone Miner. Res. 15:1477-1488. 